\title{
THE DIRICHLET ELLIPTIC PROBLEM INVOLVING REGIONAL FRACTIONAL LAPLACIAN
}

\author{
HUYUAN CHEN ${ }^{1}$ \\ Department of Mathematics, Jiangxi Normal University, \\ Nanchang, Jiangxi 330022, PR China
}

\begin{abstract}
In this paper, we study the solutions for elliptic equations involving regional fractional Laplacian

$$
\left\{\begin{aligned}
(-\Delta)_{\Omega}^{\alpha} u=f & \text { in } \quad \Omega, \\
u=g & \text { on } \quad \partial \Omega,
\end{aligned}\right.
$$

where $\Omega$ is a bounded open domain in $\mathbb{R}^{N}(N \geq 2)$ with $C^{2}$ boundary $\partial \Omega$, $\alpha \in\left(\frac{1}{2}, 1\right)$ and the operator $(-\Delta)_{\Omega}^{\alpha}$ denotes the regional fractional Laplacian. We prove that when $g \equiv 0$, problem (1) admits a unique weak solution in the cases that $f \in L^{2}(\Omega), f \in L^{1}\left(\Omega, \rho^{\beta} d x\right)$ and $f \in \mathcal{M}\left(\Omega, \rho^{\beta}\right)$, here $\rho(x)=$ $\operatorname{dist}(x, \partial \Omega), \beta=2 \alpha-1$ and $\mathcal{M}\left(\Omega, \rho^{\beta}\right)$ is a space of all Radon measures $\nu$ satisfying $\int_{\Omega} \rho^{\beta} d|\nu|<+\infty$. Finally, we provide an Integral by Parts Formula for the classical solution of (1) with general boundary data $g$.
\end{abstract}

\section{INTRODUCTION}

The usual Laplaciain operator may be thought as a macroscopic manifestation of the Brownian motion, as known from the Fokker-Plank equation for a stochastic differential equation with a Brownian motion (a Gaussian process), whereas the fractional Laplacian operator $(-\Delta)^{\alpha}$ is associated with a $2 \alpha$-stable Lévy motion (a non-Gaussian process) $L_{t}^{2 \alpha}$, $\alpha \in(0,1)$, (see [11] for a discussion about this microscopic-macroscopic relation). From the observations and experiments related to Lévy flights $([3,17,19,21])$, the fractional Laplacian described that a particle could have infinite jumps in an arbitrary time interval with intensity proportional to $\frac{1}{|x-y|^{N+2 \alpha}}$, but if the particle jumping is forced to restrict only from one point $x \in \Omega$, a bounded open domain $\Omega$ in $\mathbb{R}^{N}$, to another point $y \in \Omega$ with the same intensity, then the related process is called the censored stable process and its generator is the regional fractional Laplacian defined in $\Omega$, see the references $[5,6,16]$. In particular, the authors in [3] pointed out that the censored $2 \alpha$-stable process is conservative and will never approach $\partial \Omega$ when $\alpha \in\left(0, \frac{1}{2}\right]$ and for $\alpha \in\left(\frac{1}{2}, 1\right)$ that process could approach to the boundary $\partial \Omega$. This indicates that the Dirichlet problem involving the regional fractional Laplacian is well defined for $\alpha \in\left(\frac{1}{2}, 1\right)$ and in this note, we pay our attentions on the solutions to related Dirichlet elliptic problem with $\alpha \in\left(\frac{1}{2}, 1\right)$.

Throughout this paper, we assume that $\alpha \in\left(\frac{1}{2}, 1\right), \beta=2 \alpha-1, \Omega$ is a bounded open domain in $\mathbb{R}^{N}(N \geq 2)$ with $C^{2}$ boundary $\partial \Omega$ and $\rho(x)=\operatorname{dist}(x, \partial \Omega)$. Denote by $(-\Delta)_{\Omega}^{\alpha}$ the regional fractional Laplacian

$$
(-\Delta)_{\Omega}^{\alpha} u(x)=\lim _{\varepsilon \rightarrow 0^{+}}(-\Delta)_{\Omega, \varepsilon}^{\alpha} u(x)
$$

${ }^{1}$ chenhuyuan@yeah.net

AMS Subject Classifications: 35R11, 35D30, 35J61.

Key words: Regional Fractional Laplacian, Green Kernel, Weak solution. 
with

$$
(-\Delta)_{\Omega, \varepsilon}^{\alpha} u(x)=-c_{N, \alpha} \int_{\Omega \backslash B_{\varepsilon}(x)} \frac{u(z)-u(x)}{|z-x|^{N+2 \alpha}} d z,
$$

where $c_{N, \alpha}>0$ coincides the normalized constant of the fractional Laplacian. The main objective of this note is to study the weak solution of elliptic problem

$$
\left\{\begin{aligned}
(-\Delta)_{\Omega}^{\alpha} u=f & \text { in } \quad \Omega, \\
u=0 & \text { on } \partial \Omega
\end{aligned}\right.
$$

where $f: \Omega \rightarrow \mathbb{R}$. We will concentrate on the existence and uniqueness of the solution of (1.1) in a suitable weak sense when $f \in L^{2}(\Omega)$ or $f$ belongs to Radon measure space.

When $f \in L^{2}(\Omega)$, it involves the Hilbert space $H_{0}^{\alpha}(\Omega)$ with the scalar product

$$
\langle u, v\rangle_{\alpha}=\frac{c_{N, \alpha}}{2} \int_{\Omega} \int_{\Omega} \frac{[u(x)-u(y)][v(x)-v(y)]}{|x-y|^{N+2 \alpha}} d x d y+\int_{\Omega} u v d x, \quad \forall u, v \in H_{0}^{\alpha}(\Omega) .
$$

which is the closure of $C_{c}^{2}(\bar{\Omega})$ under the norm

$$
\|u\|_{H^{\alpha}(\Omega)}=\sqrt{\langle u, u\rangle_{\alpha}}
$$

which, shown in [10], is equivalent to the Gagliardo norm $\|u\|_{H^{\alpha}(\Omega)}$ in $H_{0}^{\alpha}(\Omega)$

$$
\|u\|_{H_{0}^{\alpha}(\Omega)}:=\left(\frac{c_{N, \alpha}}{2} \int_{\Omega} \int_{\Omega} \frac{[u(x)-u(y)]^{2}}{|x-y|^{N+2 \alpha}} d x d y\right)^{\frac{1}{2}}
$$

and its scalar product of $\|\cdot\|_{H_{0}^{\alpha}(\Omega)}$ is

$$
\langle u, v\rangle_{H_{0}^{\alpha}(\Omega)}=\frac{c_{N, \alpha}}{2} \int_{\Omega} \int_{\Omega} \frac{[u(x)-u(y)][v(x)-v(y)]}{|x-y|^{N+2 \alpha}} d x d y, \quad \forall u, v \in H_{0}^{\alpha}(\Omega) .
$$

Definition 1.1. (i) When $f \in L^{2}(\Omega)$, a function $u \in H_{0}^{\alpha}(\Omega)$, is a weak solution of (1.1), if

$$
\langle u, v\rangle_{H_{0}^{\alpha}(\Omega)}=\int_{\Omega} f(x) v(x) d x, \quad \forall v \in H_{0}^{\alpha}(\Omega) .
$$

(ii) Denote by $\mathbb{X}_{\alpha}$ the space of functions $\xi$, continuous up to the boundary, taking zero value on $\partial \Omega$ and verifying

$$
\left\|(-\Delta)_{\Omega}^{\alpha} \xi\right\|_{L^{\infty}(\Omega)}<\infty
$$

and by $\mathcal{M}\left(\Omega, \rho^{\beta}\right)$ the space of all the Radon measure $\nu$ satisfying

$$
\int_{\Omega} \rho^{\beta} d|\nu|<+\infty
$$

When $f \in \mathcal{M}\left(\Omega, \rho^{\beta}\right)$, a function $u \in L^{1}(\Omega)$ is a very weak solution of (1.1), if

$$
\int_{\Omega} u(-\Delta)_{\Omega}^{\alpha} \xi d x=\int_{\Omega} \xi d f, \quad \forall \xi \in \mathbb{X}_{\alpha}(\Omega)
$$

We notice that $\beta=1$ if $\alpha=1$, and in this case the test functions' space corresponding to very weak solution is $C_{0}^{1,1}(\Omega)$, in which the function could be controlled by the distance function $\rho$. In the regional fractional case, the test functions space $\mathbb{X}_{\alpha}(\Omega)$ plays the same role and the function in $\mathbb{X}_{\alpha}(\Omega)$ has the decay $\rho^{\beta}$, see Lemma 2.5 below.

Now we are ready to state our main theorem on the existence and uniqueness of weak solution for problem (1.1). 
Theorem 1.1. (i) Let $f \in L^{2}(\Omega)$, then problem (1.1) has a unique weak solution $u_{f}$ such that

$$
\left\|u_{f}\right\|_{H_{0}^{\alpha}(\Omega)} \leq c_{1}\|f\|_{L^{2}(\Omega)}
$$

where $c_{1}>0$.

(ii) Let $f \in \mathcal{M}\left(\Omega, \rho^{\beta}\right)$, then problem (1.1) has a unique very weak solution $u_{f}$ such that

$$
\left\|u_{f}\right\|_{L^{1}(\Omega)} \leq c_{2}\|f\|_{\mathcal{M}\left(\Omega, \rho^{\beta}\right)}
$$

where $c_{2}>0$.

For $f \in L^{2}(\Omega)$ or $f \in \mathcal{M}\left(\Omega, \rho^{\beta}\right)$, a sequence of functions $\left\{f_{n}\right\}_{n}$ in $C^{2}(\Omega) \cap C(\bar{\Omega})$ could be chosen to converge to $f$ in $L^{2}(\Omega)$ and we prove the solution of (1.1) is approached by the classical solution of

$$
\left\{\begin{aligned}
(-\Delta)_{\Omega}^{\alpha} u & =f_{n} & & \text { in } \quad \Omega, \\
u & =0 & & \text { on } \quad \partial \Omega .
\end{aligned}\right.
$$

In this approaching process, the most important tool is the Integral by Parts formula,

$$
\begin{aligned}
\int_{\Omega} u(-\Delta)_{\Omega}^{\alpha} v d x & =\frac{c_{N, \alpha}}{2} \int_{\Omega} \int_{\Omega} \frac{[u(x)-u(y)][v(x)-v(y)]}{|x-y|^{N+2 \alpha}} d x d y \\
& =\int_{\Omega} v(-\Delta)_{\Omega}^{\alpha} u d x, \quad \forall u, v \in \mathbb{X}_{\alpha}(\Omega) .
\end{aligned}
$$

Thanks to a fractional Hardy-Sobolev inequality from [12], we also show the equivalence between the norms $\|u\|_{H^{\alpha}(\Omega)}$ and $\|\cdot\|_{H_{0}^{\alpha}(\Omega)}$ for functions in $C_{0}^{\infty}(\Omega)$.

It is known that $L^{1}\left(\Omega, \rho^{\beta} d x\right)$ is a proper subset of $\mathcal{M}\left(\Omega, \rho^{\beta}\right)$ and we abuse the notation without confusion that $d f(x)=f(x) d x$ when $f \in L^{1}\left(\Omega, \rho^{\beta} d x\right)$ in the definition of very weak solution. But the proofs of the existence of very weak solutions to (1.1) are very different for $f$ in $L^{1}\left(\Omega, \rho^{\beta} d x\right)$ and in $\mathcal{M}\left(\Omega, \rho^{\beta}\right)$. For $f \in L^{1}\left(\Omega, \rho^{\beta} d x\right)$, the very weak solution is approached directly by a Cauchy sequence in $L^{1}(\Omega)$, while in the case of $f \in \mathcal{M}\left(\Omega, \rho^{\beta}\right)$, we have to prove the approximations is uniformly bounded in $L^{1}(\Omega)$ and uniformly integrable, then Dunford-Pettis Thoerem is applied to derive the very weak solution of (1.1). The elliptic problems involving measure data with second order operators have been extensively studied in $[1,2,14,20,23]$ and the reference therein, and recently, the elliptic problems involving the fractional Laplacian have been investigated by $[7,8,9]$.

Finally, we make use of the nonlocal characteristic property to build an Integral by Parts Formula for the solution $u$ of

$$
\left\{\begin{aligned}
(-\Delta)_{\Omega}^{\alpha} u=f & \text { in } \quad \Omega \\
u=g & \text { on } \quad \partial \Omega
\end{aligned}\right.
$$

for $f \in C^{2}(\Omega) \cap C(\bar{\Omega})$ and $g \in C^{2}(\partial \Omega)$. That is,

$$
\int_{\Omega} u(-\Delta)_{\Omega}^{\alpha} \xi d x=\int_{\Omega} f(x) \xi(x) d x+\int_{\partial \Omega} \frac{\partial^{\beta} \xi(x)}{\partial \vec{n}_{x}^{\beta}} g(x) d \omega(x), \quad \forall \xi \in \mathbb{X}_{\alpha}(\Omega) \cap \mathbb{D}_{\beta},
$$

where $\vec{n}_{x}$ is the unit exterior normal vector of $\Omega$ at point $x \in \partial \Omega$ and

$$
\mathbb{D}_{\beta}=\bigcup_{\tau \geq \beta}\left\{\phi_{1} \rho^{\tau}+\phi_{2}: \phi_{1}, \phi_{2} \in C^{2}(\bar{\Omega})\right\} \text {. }
$$

Here we note that

$$
\frac{\partial^{\beta} \xi(x)}{\partial \vec{n}_{x}^{\beta}}=\lim _{t \rightarrow 0^{+}} \frac{\xi(x)-\xi\left(x-t \vec{n}_{x}\right)}{t^{\beta}}=-\lim _{t \rightarrow 0^{+}} \frac{\xi\left(x-t \vec{n}_{x}\right)}{t^{\beta}}
$$


and an Integral by Parts Formula provided in [15] states as follows

$$
\int_{\Omega} w(-\Delta)_{\Omega}^{\alpha} v d x=\int_{\Omega} v(-\Delta)_{\Omega}^{\alpha} w d x, \quad \forall w, v \in \mathbb{D}_{\beta} .
$$

The paper is organized as follows. In Section $\S 2$, we study the solutions of (1.1) with $f \in$ $L^{\infty}$, including the existence and uniqueness of classical solution, the boundary regularities and also provides some important estimates for proving (1.4). Section $\S 3$ is devoted to give an Integration by Parts Formula for $u, v \in \mathbb{X}_{\alpha}(\Omega)$, then we obtain the existence and uniqueness of weak solution of (1.1) with zero boundary data and $f \in L^{2}(\Omega)$. In Section $\S 4$, we prove the very weak solution of (1.1) for $f \in L^{1}\left(\Omega, \rho^{\beta} d x\right)$ and $f \in \mathcal{M}\left(\Omega, \rho^{\beta}\right)$. Finally, we provide Integral by Parts Formula (1.6) for the solution of (1.1) with general boundary data in Section $\S 5$.

\section{Preliminary}

The purpose of this section is to introduce some preliminaries on the classical solution of (1.1). We start it by the Comparison Principle. In what follows, we denote by $c_{i}$ a generic positive constant.

Lemma 2.1. Assume that $g$ is continuous on $\partial \Omega$ and $f_{i}: \Omega \rightarrow \mathbb{R}$ with $i=1,2$ are continuous functions satisfying

$$
f_{1} \geq f_{2} \quad \text { in } \Omega .
$$

Let $u_{1}$ and $u_{2}$ be the solutions of (1.5) with $f=f_{1}$ and $f_{2}$, respectively. Then

$$
u_{1} \geq u_{2} \quad \text { in } \Omega \text {. }
$$

Furthermore, if $f \equiv 0, g \equiv 0$, then problem (1.5) only has zero solution.

Proof. By contradiction, if (2.1) fails, denoting $w=u_{1}-u_{2}$, there exists $x_{0} \in \Omega$ such that

$$
w\left(x_{0}\right)=u_{1}\left(x_{0}\right)-u_{2}\left(x_{0}\right)=\min _{x \in \Omega} w(x)<0 .
$$

Combining with $w=0$ on $\partial \Omega$, we observe that

$$
(-\Delta)_{\Omega}^{\alpha} w\left(x_{0}\right)=\int_{\Omega} \frac{w\left(x_{0}\right)-w(y)}{\left|x_{0}-y\right|^{N+2 \alpha}} d y<0 .
$$

But

$$
(-\Delta)_{\Omega}^{\alpha} w\left(x_{0}\right)=f_{1}\left(x_{0}\right)-f_{2}\left(x_{0}\right) \geq 0,
$$

which is impossible.

Our main aim here is give the regularity up to the boundary of the solution of (1.1).

Theorem 2.1. Assume that $\alpha \in\left(\frac{1}{2}, 1\right), f \in L^{\infty}(\Omega), f_{ \pm}=\max \{ \pm f, 0\}$ and $\rho(x)=$ $\operatorname{dist}(x, \partial \Omega)$. Then problem (1.1) admits a unique solution $u_{f}$ such that

$$
-c_{3}\left\|f_{-}\right\|_{L^{\infty}(\Omega)} \rho(x)^{\beta} \leq u_{f}(x) \leq c_{3}\left\|f_{+}\right\|_{L^{\infty}(\Omega)} \rho(x)^{\beta} \quad x \in \Omega .
$$

Moreover,

(i) for $\theta \in(0,2 \alpha)$ and an open set $\mathcal{O}$ satisfying $d_{\mathcal{O}}:=\operatorname{dist}(\mathcal{O}, \partial \Omega)>0$, there exists $c_{4}>0$ dependent of $d_{\mathcal{O}}$ and $\theta$ such that

$$
\left\|u_{f}\right\|_{C^{\theta}(\mathcal{O})} \leq c_{4}\|f\|_{L^{\infty}(\Omega)}
$$

(ii) there exists $c_{5}>0$ independent of $f$ such that

$$
\left\|u_{f}\right\|_{C^{\beta}(\bar{\Omega})} \leq c_{5}\|f\|_{L^{\infty}(\Omega)} .
$$


In order to consider (1.1), we need the following uniformly estimates. Denote by $G_{\Omega, \alpha}$ the Green kernel of $(-\Delta)_{\Omega}^{\alpha}$ in $\Omega \times \Omega$ and by $\mathbb{G}_{\Omega, \alpha}[\cdot]$ the Green operator defined as

$$
\mathbb{G}_{\Omega, \alpha}[f](x)=\int_{\Omega} G_{\Omega, \alpha}(x, y) f(y) d y .
$$

Lemma 2.2. Let $\alpha \in\left(\frac{1}{2}, 1\right)$ and $f \in L^{\infty}(\Omega)$, then $\mathbb{G}_{\Omega, \alpha}[f]$ is the unique solution of problem (1.1) and

$$
\left|\mathbb{G}_{\Omega, \alpha}[f](x)\right| \leq c_{6} \rho(x)^{\beta}, \quad \forall x \in \Omega .
$$

Proof. The uniqueness follows by Lemma 2.1. We observe that $\mathbb{G}_{\Omega, \alpha}[f]$ is a solution of $(-\Delta)_{\Omega}^{\alpha} w=f$ in $\Omega$ and for any $(x, y) \in \Omega \times \Omega$ with $x \neq y$,

$$
G_{\Omega, \alpha}(x, y) \leq c_{7} \min \left\{\frac{1}{|x-y|^{N-2 \alpha}}, \frac{\rho(x)^{\beta} \rho(y)^{\beta}}{|x-y|^{N-1+\beta}}\right\}
$$

see [5]. Then we have that

$$
\begin{aligned}
\left|\mathbb{G}_{\Omega, \alpha}[f](x)\right| & \leq c_{7} \int_{\Omega} \frac{\rho(x)^{\beta} \rho(y)^{\beta}}{|x-y|^{N-1+\beta}}|f(y)| d y \\
& \leq c_{7} \rho(x)^{\beta}\|f\|_{L^{\infty}(\Omega)} \int_{\Omega} \frac{\rho(y)^{\beta}}{|x-y|^{N-1+\beta}} d y \\
& \leq c_{8}\|f\|_{L^{\infty}(\Omega)} \rho(x)^{\beta}, \quad \forall x \in \Omega .
\end{aligned}
$$

Hence $\mathbb{G}_{\Omega, \alpha}[f]$ is a solution of (1.1) verifying (2.5).

In what follows, we denote

$$
u_{f}=\mathbb{G}_{\Omega, \alpha}[f] .
$$

Lemma 2.3. For any $x_{0} \in \Omega$ and $\theta \in(0,2 \alpha)$, there exists $c_{9}>0$ independent of $\rho\left(x_{0}\right)$ such that

$$
\left\|u_{f}\right\|_{C^{\theta}\left(B_{\rho_{0}}\left(x_{0}\right)\right)} \leq c_{9} \rho_{0}^{\beta-\theta}\|f\|_{L^{\infty}(\Omega)}
$$

where $\rho_{0}=\rho\left(x_{0}\right) / 3$.

Proof. For $x_{0} \in \Omega$, we denote that $\Omega_{0}=\left\{y \in \mathbb{R}^{N}: x_{0}+\rho_{0} y \in \Omega\right\}$ and

$$
v_{f}(x)=u_{f}\left(x_{0}+\rho_{0} x\right), \quad \forall x \in \mathbb{R}^{N},
$$

then by Lemma 2.2, we have that

$$
\left\|v_{f}\right\|_{L^{\infty}\left(B_{2}(0)\right)}=\left\|u_{f}\right\|_{L^{\infty}\left(B_{2 \rho_{0}}\left(x_{0}\right)\right)} \leq c_{6}\|f\|_{L^{\infty}(\Omega)} \rho_{0}^{\beta}
$$

and for $x \in B_{2}(0)$,

$$
\begin{aligned}
(-\Delta)_{\Omega_{0}}^{\alpha} v_{f}(x) & =\text { P.V.c. } c_{N, \alpha} \int_{\Omega_{0}} \frac{u_{f}\left(x_{0}+\rho_{0} x\right)-u_{f}\left(x_{0}+\rho_{0} y\right)}{|x-y|^{N+2 \alpha}} d y \\
& =\rho_{0}^{2 \alpha}(-\Delta)_{\Omega}^{\alpha} u_{f}\left(x_{0}+\rho_{0} x\right)=\rho_{0}^{2 \alpha} f\left(x_{0}+\rho_{0} x\right)
\end{aligned}
$$

and

$$
\begin{aligned}
(-\Delta)^{\alpha} v_{f}(x) & =(-\Delta)_{\Omega_{0}}^{\alpha} v_{f}(x)+v_{f}(x) \phi_{0}(x) \\
& =\rho_{0}^{2 \alpha} f\left(x_{0}+\rho_{0} x\right)+v_{f}(x) \phi_{0}(x), \quad \forall x \in B_{2}(0),
\end{aligned}
$$

where $\phi_{0}(x)=c_{N, \alpha} \int_{\mathbb{R}^{N} \backslash \Omega_{0}} \frac{1}{|x-y|^{N+2 \alpha}} d y$. Since $B_{3}(0) \subset \Omega_{0}$, we have that

$$
\phi_{0}(x) \leq c_{N, \alpha}, \quad \forall x \in B_{2}(0) .
$$


Then by [22, Proposition 2.3], we have that

$$
\begin{aligned}
\left\|v_{f}\right\|_{C^{\gamma}\left(B_{1}(0)\right)} & \leq c_{10}\left(\left\|\rho_{0}^{2 \alpha} f\left(x_{0}+\rho_{0} \cdot\right)+v_{f} \phi_{0}\right\|_{L^{\infty}\left(B_{2}(0)\right)}+\left\|v_{f}\right\|_{L^{\infty}\left(B_{2}(0)\right)}\right) \\
& \leq c_{10}\left(\rho_{0}^{2 \alpha}\|f\|_{L^{\infty}(\Omega)}+\left\|v_{f}\right\|_{L^{\infty}\left(B_{2}(0)\right)}\right) .
\end{aligned}
$$

Since

$$
\left\|v_{f}\right\|_{L^{\infty}\left(B_{2}(0)\right)}=\left\|u_{f}\right\|_{L^{\infty}\left(B_{2 \rho_{0}}\left(x_{0}\right)\right)} \leq c_{11} \rho_{0}^{\beta}\|f\|_{L^{\infty}(\Omega)},
$$

then we have that

The proof ends.

$$
\left\|u_{f}\right\|_{C^{\theta}\left(B_{\rho_{0}}\left(x_{0}\right)\right)} \leq c_{12} \rho_{0}^{\beta-\theta}\|f\|_{L^{\infty}(\Omega)} .
$$

Lemma 2.4. Let

$$
\phi(x)=\int_{\mathbb{R}^{N} \backslash \Omega} \frac{1}{|x-y|^{N+2 \alpha}} d y,
$$

then $\phi \in C_{\mathrm{loc}}^{0,1}(\Omega)$ and for some $c_{13}>1$

$$
\frac{1}{c_{13}} \rho(x)^{-2 \alpha} \leq \phi(x) \leq c_{13} \rho(x)^{-2 \alpha}, \quad \forall x \in \Omega .
$$

Proof. For $x_{1}, x_{2} \in \Omega$ and any $z \in \mathbb{R}^{N} \backslash \Omega$, we have that

$$
\left|z-x_{1}\right| \geq \rho\left(x_{1}\right)+\rho(z), \quad\left|z-x_{2}\right| \geq \rho\left(x_{2}\right)+\rho(z)
$$

and

$$
|| z-\left.x_{1}\right|^{N+2 \alpha}-\left|z-x_{2}\right|^{N+2 \alpha}\left|\leq c_{14}\right| x_{1}-x_{2} \mid\left(\left|z-x_{1}\right|^{N+2 \alpha-1}+\left|z-x_{2}\right|^{N+2 \alpha-1}\right),
$$

for some $c_{14}>0$ independent of $x_{1}$ and $x_{2}$. Then

$$
\begin{aligned}
& \left|\phi\left(x_{1}\right)-\phi\left(x_{2}\right)\right| \leq \int_{\mathbb{R}^{N} \backslash \Omega} \frac{|| z-\left.x_{2}\right|^{N+2 \alpha}-\left|z-x_{1}\right|^{N+2 \alpha} \mid}{\left|z-x_{1}\right|^{N+2 \alpha}\left|z-x_{2}\right|^{N+2 \alpha}} d z \\
& \leq c_{14}\left|x_{1}-x_{2}\right|\left[\int_{\mathbb{R}^{N} \backslash \Omega} \frac{d z}{\left|z-x_{1}\right|\left|z-x_{2}\right|^{N+2 \alpha}}+\int_{\mathbb{R}^{N} \backslash \Omega} \frac{d z}{\left|z-x_{1}\right|^{N+2 \alpha}\left|z-x_{2}\right|}\right] .
\end{aligned}
$$

By direct computation, we have that

$$
\begin{aligned}
\int_{\mathbb{R}^{N} \backslash \Omega} \frac{1}{\left|z-x_{1}\right|\left|z-x_{2}\right|^{N+2 \alpha}} d z \leq & \int_{\mathbb{R}^{N} \backslash B_{\rho\left(x_{1}\right)}\left(x_{1}\right)} \frac{1}{\left|z-x_{1}\right|^{N+2 \alpha+1}} d z \\
& +\int_{\mathbb{R}^{N} \backslash B_{\rho\left(x_{2}\right)}\left(x_{2}\right)} \frac{1}{\left|z-x_{2}\right|^{N+2 \alpha+1}} d z \\
\leq & c_{15}\left[\rho\left(x_{1}\right)^{-1-2 \alpha}+\rho\left(x_{2}\right)^{-1-2 \alpha}\right]
\end{aligned}
$$

and similar to obtain that

$$
\int_{\mathbb{R}^{N} \backslash \Omega} \frac{1}{\left|z-x_{1}\right|^{N+2 \alpha}\left|z-x_{2}\right|} d z \leq c_{15}\left[\rho\left(x_{1}\right)^{-1-2 \alpha}+\rho\left(x_{2}\right)^{-1-2 \alpha}\right] .
$$

Then

that is, $\phi$ is $C^{0,1}$ locally in $\Omega$.

$$
\left|\phi\left(x_{1}\right)-\phi\left(x_{2}\right)\right| \leq c_{14} c_{15}\left[\rho\left(x_{1}\right)^{-1-2 \alpha}+\rho\left(x_{2}\right)^{-1-2 \alpha}\right]\left|x_{1}-x_{2}\right|,
$$

We next prove (2.9). Without loss of generality, we may assume that $0 \in \partial \Omega$, the inside pointing normal vector at 0 is $e_{N}=(0, \cdots, 0,1) \in \mathbb{R}^{N}$ and let $s \in\left(0, \frac{1}{4}\right)$ such that $\mathbb{R}^{N} \backslash \Omega \subset \mathbb{R}^{N} \backslash B_{s}\left(s e_{N}\right)$ and for $t>0$, we denote the cone

$$
A_{t}=\left\{y=\left(y^{\prime}, y_{N}\right) \in \mathbb{R}^{N}: y_{N} \leq s-t\left|y^{\prime}\right|\right\} .
$$


We observe that there is $c_{0}>0$ such that

$$
\left[A_{t_{0}} \cap\left(B_{1}\left(s e_{N}\right) \backslash B_{2 s}\left(s e_{N}\right)\right)\right] \subset \mathbb{R}^{N} \backslash \Omega .
$$

By the definition of $\phi$, we have that

$$
\phi\left(s e_{N}\right)=\int_{\mathbb{R}^{N} \backslash \Omega} \frac{1}{\left|s e_{N}-y\right|^{N+2 \alpha}} d y \leq \int_{\mathbb{R}^{N} \backslash B_{s}\left(s e_{N}\right)} \frac{1}{\left|s e_{N}-y\right|^{N+2 \alpha}} d y \leq c_{16} s^{-2 \alpha} .
$$

On the other hand, we have that

$$
\int_{\mathbb{R}^{N} \backslash \Omega} \frac{1}{\left|s e_{N}-y\right|^{N+2 \alpha}} d y \geq \int_{A_{c_{0}} \cap\left(B_{1}\left(s e_{N}\right) \backslash B_{2 s}\left(s e_{N}\right)\right)} \frac{1}{\left|s e_{N}-y\right|^{N+2 \alpha}} d y \geq c_{17} s^{-2 \alpha} .
$$

The proof ends.

Now we are ready to prove Theorem 2.1.

Proof of Theorem 2.1. By Lemma 2.2, $u_{f}$ is the unique solution of (1.1). Since $\mathbb{G}_{\Omega, \alpha}\left[f_{+}\right]$, $-\mathbb{G}_{\Omega, \alpha}\left[f_{-}\right]$are solutions of (1.1) replaced $f$ by $f_{+}$and $f_{-}$respectively. Then (2.2) follows by Lemma 2.1.

Proof of (2.3). Let $\tilde{w}=w$ in $\Omega, \tilde{w}=0$ in $\mathbb{R}^{N} \backslash \bar{\Omega}$, we observe that

$$
(-\Delta)^{\alpha} \tilde{w}(x)=(-\Delta)_{\Omega}^{\alpha} w(x)+w(x) \phi(x), \quad \forall x \in \Omega,
$$

where $\phi$ is defined by (2.8). It follows by Lemma $2.4, \phi \in C_{\text {loc }}^{0,1}(\Omega)$. Then

$$
(-\Delta)^{\alpha} \tilde{w}(x)=f(x)+w(x) \phi(x), \quad \forall x \in \Omega .
$$

Let $\mathcal{O}_{1}$ be a $C^{2}$ open set such that

$$
\mathcal{O} \subset \mathcal{O}_{1}, \quad \operatorname{dist}\left(\mathcal{O}_{1}, \partial \Omega\right)=d_{\mathcal{O}} / 2, \quad \text { and } \operatorname{dist}\left(\mathcal{O}, \partial \mathcal{O}_{1}\right)=d_{\mathcal{O}} / 2 .
$$

By $[9$, Lemma 3.1], for any $\theta \in(0,2 \alpha)$, we have that

$$
\|\tilde{w}\|_{C^{\theta}(\mathcal{O})} \leq c_{18}\left[\|\tilde{w}\|_{L^{\infty}\left(\mathcal{O}_{1}\right)}+\|\tilde{w}\|_{L^{1}(\Omega)}+\|f+\tilde{w} \phi\|_{L^{\infty}\left(\mathcal{O}_{1}\right)}\right] .
$$

By Lemma 2.2 and Lemma 2.4, we obtain that

$$
\|\tilde{w}\|_{L^{\infty}\left(\mathcal{O}_{1}\right)}+\|\tilde{w}\|_{L^{1}(\Omega)} \leq\|f\|_{L^{\infty}(\Omega)}
$$

and

$$
|\tilde{w}(x)| \phi(x) \leq c_{19} \rho(x)^{2 \alpha-1}\|f\|_{L^{\infty}(\Omega)} \rho(x)^{-2 \alpha} \leq c_{19} \rho(x)^{-1}\|f\|_{L^{\infty}(\Omega)},
$$

then

$$
\|f+\tilde{w} \phi\|_{L^{\infty}\left(\mathcal{O}_{1}\right)} \leq\|f\|_{L^{\infty}(\Omega)}+\|\tilde{w} \phi\|_{L^{\infty}\left(\mathcal{O}_{1}\right)} \leq c_{20} d_{\mathcal{O}}^{-1}\|f\|_{L^{\infty}(\Omega)} .
$$

Then (2.3) holds.

Proof of (2.4). Taking $\theta=2 \alpha-1$ in Lemma 2.3, we have that

$$
\frac{u(x)-u(y)}{|x-y|^{\theta}} \leq c_{21}\|f\|_{L^{\infty}(\Omega)}
$$

for all $x, y$ such that $y \in B_{R}(x)$ with $R=\rho(x) / 3$. We next show that (2.11) holds for all $x, y \in \bar{\Omega}$ with some renewed constant.

Indeed, we observe that after a Lipschitz change of coordinates, the bound (2.11) remains the same except for the value of the constant $c$. Then we can flatten the boundary near $x_{0} \in \partial \Omega$ to assume that $\Omega \cap B_{\rho_{0}}\left(x_{0}\right)=\left\{x_{n}>0\right\} \cap B_{1}(0)$. Thus, (2.11) holds for all $x, y$ satisfying $|x-y| \leq \gamma x_{n}$ for some $\gamma=\gamma(\Omega) \in(0,1)$ dependent of the Lipschitz mapping.

Let $z=\left(z^{\prime}, z_{n}\right)$ and $w=\left(w^{\prime}, w_{n}\right)$ be two points in $\left\{x_{n}>0\right\} \cap B_{1 / 4}(0)$ and $r=|z-w|$. Denote that $\bar{z}=\left(z^{\prime}, z_{n}+r\right), \bar{w}=\left(w^{\prime}, w_{n}+r\right)$ and $z_{k}=\left(1-\gamma^{k}\right) z+\gamma^{k} \bar{z}$ and $w_{k}=$ 
$\gamma^{k} w+\left(1-\gamma^{k}\right) \bar{w}, k \geq 0$. Then, using the bound (2.11) whenever $|x-y| \leq \gamma x_{n}$, we have that

$$
\left|u\left(z_{k+1}\right)-u\left(z_{k}\right)\right| \leq c_{22}\left|z_{k+1}-z_{k}\right|^{\theta}=c_{21}\left|\gamma^{k}(z-\bar{z})(\gamma-1)\right|^{\theta} \leq c_{22} \gamma^{k}|z-\bar{z}|
$$

Moreover, since $x_{n}>r$ in all the segment joining $\bar{z}$ and $\bar{w}$, splitting this segment into a bounded number of segments of length less than $\gamma r$, we obtain that

$$
|u(\bar{z})-u(\bar{w})| \leq c_{23}|\bar{z}-\bar{w}|^{\theta} \leq c_{23} r^{\theta} .
$$

Therefore,

$$
\begin{aligned}
|u(z)-u(w)| & \leq \sum_{k \geq 0}\left|u\left(z_{k+1}\right)-u\left(z_{k}\right)\right|+|u(\bar{z})-u(\bar{w})|+\sum_{k \geq 0}\left|u\left(w_{k+1}\right)-u\left(w_{k}\right)\right| \\
& \leq\left(c_{24} \sum_{k \geq 0}\left(\gamma^{k} r\right)^{\theta}+c_{25} r^{\theta}\right)\left(\|u\|_{L^{\infty}\left(\mathbb{R}^{N}\right)}+\|g\|_{L^{\infty}(\Omega)}\right) \\
& \leq c_{26}\left(\|u\|_{L^{\infty}\left(\mathbb{R}^{N}\right)}+\|g\|_{L^{\infty}(\Omega)}\right)|z-w|^{\theta},
\end{aligned}
$$

which ends the proof.

For a unbounded nonhomogeneous term $f$, we have that

Lemma 2.5. Assume that $f$ is a $C_{l o c}^{\gamma}(\Omega)$ function satisfying

$$
|f(x)| \leq c_{27} \rho(x)^{-\beta}, \quad \forall x \in \Omega,
$$

where $\gamma \in(0,1)$. Then problem (1.1) has a unique solution $u_{f}$ satisfying

$$
\left|u_{f}(x)\right| \leq c_{28}\left\|f \rho^{\beta}\right\|_{L^{\infty}(\Omega)} \rho^{\beta}(x), \quad \forall x \in \Omega .
$$

Proof. The uniqueness follows by Lemma 2.1. It is known that $\mathbb{G}_{\Omega, \alpha}[f]$ is a solution of $(-\Delta)_{\Omega}^{\alpha} w=f$ in $\Omega$. From (2.6), we have that for $x \in \Omega$,

$$
\begin{aligned}
\left|\mathbb{G}_{\Omega, \alpha}[f](x)\right| & \leq c_{7} \int_{\Omega} \frac{\rho(x)^{\beta} \rho(y)^{\beta}}{|x-y|^{N-2+2 \alpha}}|f(y)| d y \\
& \leq c_{7} \rho(x)^{\beta}\left\|f \rho^{\beta}\right\|_{L^{\infty}(\Omega)} \int_{\Omega} \frac{1}{|x-y|^{N-2+2 \alpha}} d y \\
& \leq c_{7}\left\|f \rho^{\beta}\right\|_{L^{\infty}(\Omega)} \rho(x)^{\beta} \int_{B_{d_{0}}(x)} \frac{1}{|x-y|^{N-2+2 \alpha}} d y,
\end{aligned}
$$

where $d_{0}=\sup _{x, y \in \Omega}|x-y|$ and $\int_{B_{d_{0}}(x)} \frac{1}{|x-y|^{N-2+2 \alpha}} d y<+\infty$ by the fact that $N-2+2 \alpha<N$. Therefore, we obtain that $\mathbb{G}_{\Omega, \alpha}[f]$ is a solution of (1.1) satisfying (2.12).

Remark 2.1. We remark that (2.12) holds for $v \in \mathbb{X}_{\alpha}(\Omega)$. In fact, let $f=(-\Delta)_{\Omega}^{\alpha} v$, which satisfies

$$
\left\|f \rho^{\beta}\right\|_{L^{\infty}(\Omega)}<+\infty
$$

The next proposition plays an important role in the proof of Integration by Parts Formula with nonzero Dirichlet boundary condition. For this purpose, we introduce some notations. Denote

$$
\Omega_{\delta}:=\{x \in \Omega: \rho(x)>\delta\} \text { and } A_{\delta}:=\{x \in \Omega: \rho(x)<\delta\} .
$$

Since $\Omega$ is $C^{2}$, there exists $\delta_{0}>0$ such that $\Omega_{\delta}$ is $C^{2}$ for any $\delta \in\left(0, \delta_{0}\right]$ and it is known that for any $x \in \partial \Omega_{\delta}$, there exists $x^{*} \in \partial \Omega$ such that

$$
\left|x-x^{*}\right|=\rho(x) \quad \text { and } \quad x=x^{*}+\rho(x) \vec{n}_{x^{*}} .
$$


Proposition 2.1. Assume that $f \in C^{2}(\Omega) \cap C(\bar{\Omega})$ and $g \in C^{2}(\partial \Omega)$. Let $u$ be the classical solution of (1.5). Then $u \in C^{2}(\Omega) \cap C^{\beta}(\bar{\Omega})$. Furthermore, for $\delta \in\left(0, \delta_{0}\right)$, there exists $c_{29}>0$ such that

$$
|u(x)-u(y)|<c_{29} \rho(x)^{\beta-1}|x-y|, \quad \forall x \in \Omega_{\delta}, \forall y \in A_{\delta} .
$$

Proof. To prove $u \in C^{2}(\Omega) \cap C^{\beta}(\bar{\Omega})$. Here we only have to prove $u \in C^{2}(\Omega) \cap C^{\beta}(\bar{\Omega})$ in the case that $g \equiv 0$. In fact, since $\Omega$ is $C^{2}$ and $g \in C^{2}(\partial \Omega)$, then there exists $G \in C^{2}(\bar{\Omega})$ such that

$$
G=g \quad \text { on } \partial \Omega \text {. }
$$

Now we only consider the regularity of $u-G$, which is the solution of

$$
\begin{aligned}
(-\Delta)_{\Omega}^{\alpha} u & =f-(-\Delta)_{\Omega}^{\alpha} G & & \text { in } \quad \Omega, \\
u & =0 & & \text { on } \partial \Omega .
\end{aligned}
$$

So it follows by Theorem 2.1 that $u \in C^{\beta}(\bar{\Omega})$.

We next prove $u \in C^{2}(\Omega)$. Extend the function $u$ by zero in $\mathbb{R}^{N} \backslash \Omega$, still denote it $u$, and then

$$
(-\Delta)^{\alpha} u(x)=(-\Delta)_{\Omega}^{\alpha} u(x)+u(x) \phi(x)=f(x)+u(x) \phi(x), \quad \forall x \in \Omega,
$$

where $(-\Delta)^{\alpha}$ is the global fractional Laplacian. From Lemma 2.4, $\phi \in C_{l o c}^{0,1}(\Omega)$, applying [22, Corollary 1.6] with $\theta<1+2 \alpha$, we have that

$$
u \in C_{l o c}^{\theta}(\Omega) \text {. }
$$

This means $u \in C^{2}(\Omega)$ since we can choose $\theta>2$.

To prove (2.14). By the compactness of $\partial \Omega$, we only consider a point $x_{0} \in \partial \Omega$ and for simplicity, we can assume that $x_{0}=0$. Let $x=t \vec{n}_{0}$ with $t \in(0, \delta)$ and $y \in B_{\frac{\delta}{3}}\left(\delta \vec{n}_{0}\right) \cap \Omega_{\delta}$, for any $t \in(0, \delta]$, there exists $n$ depending on $t$ such that

$$
t \in\left(\frac{\delta}{3^{n+1}}, \frac{\delta}{3^{n}}\right)
$$

and then we choose $x_{k}=x+\frac{1}{3^{k}}(y-x), k=0,1, \cdots, n$. We observe that

$$
\rho\left(x_{k}\right) \geq \frac{\delta}{3^{k}}, \quad k=0,1, \cdots, n .
$$

From Lemma 2.3, it follows that for $k=0,1, \cdots, n$

$$
\|w\|_{C^{0,1}\left(B_{\rho\left(x_{k}\right) / 2}\left(x_{k}\right)\right)} \leq c_{30} \rho\left(x_{k}\right)^{\beta-1}\|f\|_{L^{\infty}(\Omega)} \leq c_{30}\left(\frac{\delta}{3^{k}}\right)^{\beta-1}\|f\|_{L^{\infty}(\Omega)} .
$$

It is obvious that $x_{0}=y$ and

$$
\left|x_{k}-x_{k+1}\right|<\frac{|x-y|}{3^{k}} .
$$

Then we have that for $k=0,1, \cdots, n$,

$$
\begin{aligned}
\left|w\left(x_{k}\right)-w\left(x_{k-1}\right)\right| & \leq\|w\|_{C^{0,1}\left(B_{\frac{\rho\left(x_{k}\right)}{2}}\left(x_{k}\right)\right)}\left|x_{k}-x_{k-1}\right| \\
& \leq c_{30}\left(\frac{\delta}{3^{k}}\right)^{\beta-1}\left|x_{k}-x_{k-1}\right| \\
& \leq c_{30} t^{\beta-1}\left|x_{k}-x_{k-1}\right|,
\end{aligned}
$$

therefore,

$$
\left|w(x)-w\left(x_{0}\right)\right| \leq c_{7} t^{(1-\beta)} \sum_{k=0}^{n} \frac{1}{3^{k}}|x-y| \leq c_{31} t^{\beta-1}|x-y|,
$$


where $c_{31}>0$ is independent of $t$. So for some $c_{32}>0$, we have that

$$
|w(x)-w(y)| \leq c_{32} \rho(x)^{\beta-1}|x-y| .
$$

For $y \in \Omega_{\delta} \backslash B_{\frac{\delta}{3}}\left(\delta \vec{n}_{0}\right)$, we may choose $y^{\prime} \in B_{\frac{\delta}{3}}\left(\delta \vec{n}_{0}\right) \cap \Omega_{\delta}$. There are at most $N_{0}$ points $y_{k} \in \Omega_{\delta}$ connecting $y$ and $y^{\prime}$ such that

We see that

$$
\frac{\delta}{3} \leq\left|y_{k}-y_{k-1}\right| \leq \frac{\delta}{2}
$$

$$
\left|w(y)-w\left(y^{\prime}\right)\right| \leq c_{33} \delta^{\beta-1}\left|y-y^{\prime}\right|
$$

From (2.17), we see that

$$
\left|w(x)-w\left(y^{\prime}\right)\right| \leq c_{32} \rho(x)^{\beta-1}\left|x-y^{\prime}\right| .
$$

Since $\left|y-y^{\prime}\right| \geq \frac{\delta}{3}$ and $|x-y|>\delta$, then

$$
\begin{aligned}
|w(x)-w(y)| & \leq\left|w(x)-w\left(y^{\prime}\right)\right|+\left|w(y)-w\left(y^{\prime}\right)\right| \\
& \leq c_{33} \delta^{\beta-1}\left|y-y^{\prime}\right|+c_{9} \rho(x)^{\beta-1}\left|x-y^{\prime}\right| \\
& \leq c_{34} \rho(x)^{\beta-1}|x-y| .
\end{aligned}
$$

We finish the proof.

Lemma 2.6. Assume that $f \in C^{2}(\Omega) \cap C(\bar{\Omega}), g \in C^{2}(\partial \Omega)$ and $w$ is the classical solution of (1.5). Then

$$
\int_{\Omega} \int_{\Omega} \frac{[u(x)-u(y)]^{2}}{|x-y|^{N+2 \alpha}} d x d y<+\infty
$$

Proof. From the interior regularity, we know that $u \in C^{2}(\Omega) \cap C^{\beta}(\bar{\Omega})$. From $[16$, Theorem 3.4], it infers that

$$
\begin{aligned}
\frac{c_{N, \alpha}}{2} & \int_{\Omega_{\delta}} \int_{\Omega_{\delta}} \frac{[u(x)-u(y)]^{2}}{|x-y|^{N+2 \alpha}} d x d y \\
& =\int_{\Omega_{\delta}} u(x)(-\Delta)_{\Omega_{\delta}}^{\alpha} u(x) d x \\
& =c_{N, \alpha} \int_{\Omega_{\delta}} \int_{A_{\delta}} \frac{u(x)-u(y)}{|x-y|^{N+2 \alpha}} u(x) d y d x+\int_{\Omega_{\delta}} u(x)(-\Delta)_{\Omega}^{\alpha} u(x) d x \\
& =c_{N, \alpha} \int_{\Omega_{\delta}} \int_{A_{\delta}} \frac{u(x)-u(y)}{|x-y|^{N+2 \alpha}} u(x) d y d x+\int_{\Omega_{\delta}} u(x) f(x) d x .
\end{aligned}
$$

We observe that

$$
\left|\int_{\Omega_{\delta}} u(x) f(x) d x\right| \leq|\Omega|\|u\|_{L^{\infty}(\Omega)}\|f\|_{L^{\infty}(\Omega)} .
$$

From Proposition 2.1, we derive that

$$
\begin{aligned}
\int_{\Omega_{\delta}} \int_{A_{\delta}} \frac{|u(x)-u(y)|}{|x-y|^{N+2 \alpha}}|u(x)| d y d x & \leq\|u\|_{L^{\infty}(\Omega)} \int_{A_{\delta}} \int_{\Omega_{\delta}} \frac{|u(x)-u(y)|}{|x-y|^{N+2 \alpha}} d x d y \\
& \leq c_{35}\|u\|_{L^{\infty}(\Omega)} \int_{A_{\delta}} \rho(y)^{\beta-1} \int_{\Omega_{\delta}} \frac{1}{|x-y|^{N+2 \alpha-1}} d x d y \\
& \leq c_{36}\|u\|_{L^{\infty}(\Omega)} \int_{A_{\delta}} \rho(y)^{\beta-1} \int_{\delta-\rho(y)}^{d_{0}} \frac{1}{r^{2 \alpha}} d r d y \\
& \leq c_{37}\|u\|_{L^{\infty}(\Omega)} \int_{A_{\delta}} \rho(y)^{\beta-1}(\delta-\rho(y))^{-\beta} d y .
\end{aligned}
$$


Since $\Omega$ is $C^{2}$, then for $t \in(0, \delta)$ and $\delta \leq \delta_{0}$, we have that

$$
\frac{1}{2}|\partial \Omega| \leq\left|\partial \Omega_{t}\right| \leq 2|\partial \Omega|
$$

and by Fubini's theorem

$$
\begin{aligned}
\int_{A_{\delta}} \rho(y)^{\beta-1}(\delta-\rho(y))^{-\beta} d y & =\int_{0}^{\delta} t^{\beta-1}(\delta-t)^{-\beta}\left|\partial \Omega_{t}\right| d t \\
& \leq 2|\partial \Omega| \int_{0}^{\delta} t^{\beta-1}(\delta-t)^{-\beta} d t \\
& =2|\partial \Omega| \int_{0}^{1} t^{\beta-1}(1-t)^{-\beta} d t
\end{aligned}
$$

Therefore, for some $c_{38}>0$ independent of $\delta$ there holds that

$$
\int_{\Omega_{\delta}} \int_{A_{\delta}} \frac{|u(x)-u(y)|}{|x-y|^{N+2 \alpha}}|u(x)| d y d x<c_{38}
$$

thus, together with (2.19)-(2.20), we derive that

$$
\int_{\Omega} \int_{\Omega} \frac{[u(x)-u(y)]^{2}}{|x-y|^{N+2 \alpha}} d x d y<+\infty .
$$

The proof ends.

Corollary 2.1. Assume that $f, h \in C^{2}(\Omega) \cap C(\bar{\Omega})$ and $u, w$ are the classical solution of (1.1) with nonhomogeneous nonlinearities $f$ and $h$, respectively.

Then

$$
\lim _{\delta \rightarrow 0^{+}} \int_{\Omega_{\delta}} \int_{A_{\delta}} \frac{u(x)-u(y)}{|x-y|^{N+2 \alpha}} w(y) d y d x=0 .
$$

Proof. From Theorem 2.1,

$$
|w(x)| \leq c_{15} \rho(x)^{\beta}, \quad \forall x \in \Omega .
$$

Thus,

$$
\begin{aligned}
\int_{\Omega_{\delta}} \int_{A_{\delta}} \frac{|u(x)-u(y)|}{|x-y|^{N+2 \alpha}}|w(y)| d y d x & \leq\|w\|_{L^{\infty}\left(A_{\delta}\right)} \int_{A_{\delta}} \int_{\Omega_{\delta}} \frac{|u(x)-u(y)|}{|x-y|^{N+2 \alpha}} d x d y \\
& \leq c_{15} \delta^{\beta} \int_{A_{\delta}} \rho^{\beta-1}(y)(\delta-\rho(y))^{-\beta} d y .
\end{aligned}
$$

By (2.21), we have that

$$
\int_{\Omega_{\delta}} \int_{A_{\delta}} \frac{|u(x)-u(y)|}{|x-y|^{N+2 \alpha}}|w(y)| d y d x \leq c_{38} \delta^{\beta},
$$

then (2.22) holds.

\section{ZERO BOUNDARY DATA}

3.1. Classical solution. In this subsection, we concentrate on the classical solution of (1.1) when $f \in C^{2}(\Omega) \cap C(\bar{\Omega})$.

Proposition 3.1. Assume that $f \in C^{2}(\Omega) \cap C(\bar{\Omega})$ and $u$ is the classical solution of (1.1). Then

$$
\int_{\Omega} u(-\Delta)_{\Omega}^{\alpha} v d x=\int_{\Omega} f(x) v(x) d x, \quad \forall v \in \mathbb{X}_{\alpha}(\Omega) .
$$


Proof. Let $h(x)=(-\Delta)_{\Omega}^{\alpha} v(x)$ and $h_{n}$ be a sequence of $C^{2}(\Omega) \cap C(\bar{\Omega})$ functions such that

$$
\lim _{n \rightarrow \infty}\left\|h_{n}-h\right\|_{L^{\infty}(\Omega)}=0 .
$$

Let $v_{n}$ be the solution of

$$
\left\{\begin{aligned}
(-\Delta)_{\Omega}^{\alpha} u=h_{n} & \text { in } \quad \Omega, \\
u=0 & \text { on } \quad \partial \Omega .
\end{aligned}\right.
$$

and then

$$
\left\|v_{n}\right\|_{C^{\beta}(\Omega)} \leq c_{16}\|h\|_{L^{\infty}(\Omega)} .
$$

From Lemma 2.6, we have that

$$
\int_{\Omega} \int_{\Omega} \frac{[u(x)-u(y)]^{2}}{|x-y|^{N+2 \alpha}} d x d y<+\infty \quad \text { and } \quad \int_{\Omega} \int_{\Omega} \frac{\left[v_{n}(x)-v_{n}(y)\right]^{2}}{|x-y|^{N+2 \alpha}} d x d y<+\infty,
$$

which imply that

$$
\int_{\Omega_{\delta}} \int_{\Omega_{\delta}} \frac{\left|[u(x)-u(y)]\left[v_{n}(x)-v_{n}(y)\right]\right|}{|x-y|^{N+2 \alpha}} d x d y<+\infty .
$$

From [16, Theorem 3.4], it infers that

$$
\begin{aligned}
\frac{c_{N, \alpha}}{2} & \int_{\Omega} \int_{\Omega} \frac{[u(x)-u(y)]\left[v_{n}(x)-v_{n}(y)\right]}{|x-y|^{N+2 \alpha}} d x d y \\
= & \frac{c_{N, \alpha}}{2} \lim _{\delta \rightarrow 0^{+}} \int_{\Omega_{\delta}} \int_{\Omega_{\delta}} \frac{[u(x)-u(y)]\left[v_{n}(x)-v_{n}(y)\right]}{|x-y|^{N+2 \alpha}} d x d y \\
= & \lim _{\delta \rightarrow 0^{+}} \int_{\Omega_{\delta}} v_{n}(x)(-\Delta)_{\Omega_{\delta}}^{\alpha} u(x) d x \\
= & \int_{\Omega} v_{n}(x)(-\Delta)_{\Omega}^{\alpha} u(x) d x+c_{N, \alpha} \lim _{\delta \rightarrow 0^{+}} \int_{\Omega_{\delta}} \int_{A_{\delta}} \frac{u(x)-u(y)}{|x-y|^{N+2 \alpha}} v_{n}(x) d y d x \\
= & \int_{\Omega} v_{n}(x) f(x) d x+c_{N, \alpha} \lim _{\delta \rightarrow 0^{+}} \int_{\Omega_{\delta}} \int_{A_{\delta}} \frac{[u(x)-u(y)]\left[v_{n}(x)-v_{n}(y)\right]}{|x-y|^{N+2 \alpha}} d y d x \\
& +c_{N, \alpha} \lim _{\delta \rightarrow 0^{+}} \int_{\Omega_{\delta}} \int_{A_{\delta}} \frac{u(x)-u(y)}{|x-y|^{N+2 \alpha}} v_{n}(y) d y d x
\end{aligned}
$$

and by Corollary 2.1, we have that

$$
c_{N, \alpha} \lim _{\delta \rightarrow 0^{+}} \int_{\Omega_{\delta}} \int_{A_{\delta}} \frac{[u(x)-u(y)]\left[v_{n}(x)-v_{n}(y)\right]}{|x-y|^{N+2 \alpha}} d y d x=0
$$

and

Therefore,

$$
c_{N, \alpha} \lim _{\delta \rightarrow 0^{+}} \int_{\Omega_{\delta}} \int_{A_{\delta}} \frac{u(x)-u(y)}{|x-y|^{N+2 \alpha}} v_{n}(y) d y d x=0
$$

$$
\frac{c_{N, \alpha}}{2} \int_{\Omega} \int_{\Omega} \frac{[u(x)-u(y)]\left[v_{n}(x)-v_{n}(y)\right]}{|x-y|^{N+2 \alpha}} d x d y=\int_{\Omega} v_{n}(-\Delta)_{\Omega}^{\alpha} u d x=\int_{\Omega} v_{n} f d x .
$$

Since $u$ and $v_{n}$ have the same role the above procedures, then

$$
\frac{c_{N, \alpha}}{2} \int_{\Omega} \int_{\Omega} \frac{[u(x)-u(y)]\left[v_{n}(x)-v_{n}(y)\right]}{|x-y|^{N+2 \alpha}} d x d y=\int_{\Omega} u(-\Delta)_{\Omega}^{\alpha} v_{n} d x=\int_{\Omega} h_{n} u d x .
$$

Therefore, (3.1) holds.

From the above observations, we are ready to prove the Integral by Parts Formula for the regional fractional Laplacian. 
Theorem 3.1. Let $u, v \in \mathbb{X}_{\alpha}(\Omega)$, then

$$
\int_{\Omega} \int_{\Omega} \frac{[u(x)-u(y)]^{2}}{|x-y|^{N+2 \alpha}} d x d y \leq c_{17}\left\|(-\Delta)_{\Omega}^{\alpha} u\right\|_{L^{\infty}(\Omega)}^{2}
$$

and

$$
\int_{\Omega} u(-\Delta)_{\Omega}^{\alpha} v d x=\frac{c_{N, \alpha}}{2} \int_{\Omega} \int_{\Omega} \frac{[u(x)-u(y)][v(x)-v(y)]}{|x-y|^{N+2 \alpha}} d x d y=\int_{\Omega} v(-\Delta)_{\Omega}^{\alpha} u d x .
$$

Proof. Let $f(x)=(-\Delta)_{\Omega}^{\alpha} u(x), h(x)=(-\Delta)_{\Omega}^{\alpha} v(x)$, and choose $\left\{f_{n}\right\}_{n},\left\{h_{n}\right\}_{n}$ two sequences of $C^{2}(\Omega) \cap C(\bar{\Omega})$ functions such that

$$
\lim _{n \rightarrow \infty}\left\|f_{n}-f\right\|_{L^{\infty}(\Omega)}=0 \text { and } \lim _{n \rightarrow \infty}\left\|h_{n}-h\right\|_{L^{\infty}(\Omega)}=0 .
$$

Let $u_{n}$ and $v_{n}$ be the solution of (3.2) with nonhomogeneous terms $f_{n}$ and $h_{n}$ respectively. Integrating (3.2) with nonhomogeneous terms $f_{n}$ by $u_{n}$ and $v_{n}$ over $\Omega$, from (3.3), we have that

$$
\frac{c_{N, \alpha}}{2} \int_{\Omega} \int_{\Omega} \frac{\left[u_{n}(x)-u_{n}(y)\right]^{2}}{|x-y|^{N+2 \alpha}} d x d y=\int_{\Omega} u_{n}(-\Delta)_{\Omega}^{\alpha} u_{n} d x=\int_{\Omega} u_{n} f_{n} d x
$$

and

$$
\frac{c_{N, \alpha}}{2} \int_{\Omega} \int_{\Omega} \frac{\left[u_{n}(x)-u_{n}(y)\right]\left[v_{n}(x)-v_{n}(y)\right]}{|x-y|^{N+2 \alpha}} d x d y=\int_{\Omega} v_{n}(-\Delta)_{\Omega}^{\alpha} u_{n} d x=\int_{\Omega} v_{n} f_{n} d x .
$$

Since

it infers from (3.7) that

$$
\left\|u_{n}\right\|_{C^{\beta}(\bar{\Omega})} \leq c_{18}\left\|f_{n}\right\|_{L^{\infty}(\Omega)} \leq c_{39}\left\|(-\Delta)_{\Omega}^{\alpha} u\right\|_{L^{\infty}(\Omega)},
$$

$$
\int_{\Omega} \int_{\Omega} \frac{\left[u_{n}(x)-u_{n}(y)\right]^{2}}{|x-y|^{N+2 \alpha}} d x d y \leq c_{40}\left\|(-\Delta)_{\Omega}^{\alpha} u\right\|_{L^{\infty}(\Omega)}^{2}
$$

This implies that for any $\epsilon>0$ and any $n \in \mathbb{N}$,

$$
\int_{\Omega} \int_{\Omega} \frac{\left[u_{n}(x)-u_{n}(y)\right]^{2}}{|x-y|^{N+2 \alpha}} \chi_{(\epsilon, \infty)}(|x-y|) d x d y \leq c_{41}\left\|(-\Delta)_{\Omega}^{\alpha} u\right\|_{L^{\infty}(\Omega)}^{2},
$$

passing to the limit as $n \rightarrow \infty$, then we obtain that for any $\epsilon>0$,

$$
\int_{\Omega} \int_{\Omega} \frac{[u(x)-u(y)]^{2}}{|x-y|^{N+2 \alpha}} \chi_{(\epsilon, \infty)}(|x-y|) d x d y \leq c_{41}\left\|(-\Delta)_{\Omega}^{\alpha} u\right\|_{L^{\infty}(\Omega)}^{2} .
$$

Since the left hand side of above inequality is decreasing with respective to $\epsilon>0$ and the right hand side is independent of $\epsilon$, so passing to the limit as $\epsilon \rightarrow 0^{+}$, we derive (3.4).

To prove (3.5). It is obvious that $v$ verifies (3.4). Then $\frac{u_{n}(x)-u_{n}(y)}{|x-y|^{\frac{N-2 \alpha}{2}}}$ converges to $\frac{u(x)-u(y)}{|x-y|^{\frac{N+2 \alpha}{2}}}$ in $L^{2}(\Omega \times \Omega)$ and $\frac{v_{n}(x)-v_{n}(y)}{|x-y|^{\frac{N+2 \alpha}{2}}}$ converges to $\frac{v(x)-v(y)}{|x-y|^{\frac{N+2 \alpha}{2}}}$ in $L^{2}(\Omega \times \Omega)$, thus,

$$
\lim _{n \rightarrow \infty} \int_{\Omega} \int_{\Omega} \frac{\left[u_{n}(x)-u_{n}(y)\right]\left[v_{n}(x)-v_{n}(y)\right]}{|x-y|^{N+2 \alpha}} d x d y=\int_{\Omega} \int_{\Omega} \frac{[u(x)-u(y)][v(x)-v(y)]}{|x-y|^{N+2 \alpha}} d x d y
$$

which, together with (3.6), implies that

$$
\int_{\Omega} v(-\Delta)_{\Omega}^{\alpha} u d x=\frac{c_{N, \alpha}}{2} \int_{\Omega} \int_{\Omega} \frac{[u(x)-u(y)][v(x)-v(y)]}{|x-y|^{N+2 \alpha}} d x d y .
$$

The same to conclude that

$$
\int_{\Omega} u(-\Delta)_{\Omega}^{\alpha} v d x=\frac{c_{N, \alpha}}{2} \int_{\Omega} \int_{\Omega} \frac{[u(x)-u(y)][v(x)-v(y)]}{|x-y|^{N+2 \alpha}} d x d y
$$

and (3.5) holds. 
3.2. Weak solution when $f \in L^{2}(\Omega)$. Our aim in this subsection is to consider the weak solution of (1.1) when the nonhomogeneous term $f$ is weakened from $L^{\infty}(\Omega)$ to $L^{2}(\Omega)$. To this end, we have to involve the fractional Hilbert space $H_{0}^{\alpha}(\Omega)$, which is the closure of $C_{c}^{2}(\Omega)$ under the norm of

$$
\|u\|_{H^{\alpha}(\Omega)}:=\left(\frac{c_{N, \alpha}}{2} \int_{\Omega} \int_{\Omega} \frac{[u(x)-u(y)]^{2}}{|x-y|^{N+2 \alpha}} d x d y\right)^{\frac{1}{2}}+\|u\|_{L^{2}(\Omega)} .
$$

This is called as Gagliardo norm and we denote by $\|u\|_{H_{0}^{\alpha}(\Omega)}$ the first part of (3.9) on the right hand side, which, we shall prove, is a equivalent norm of $\|u\|_{H^{\alpha}(\Omega)}$ in $H_{0}^{\alpha}(\Omega)$. Then we may say that the space $H_{0}^{\alpha}(\Omega)$ is the closure of $C_{c}^{2}(\bar{\Omega})$ under the norm $\|\cdot\|_{H_{0}^{\alpha}(\Omega)}$.

We make use of a Poincaré type inequality to prove the equivalence of the norms $\|\cdot\|_{H^{\alpha}(\Omega)}$ and $\|\cdot\|_{H_{0}^{\alpha}(\Omega)}$.

Proposition 3.2. The norms $\|\cdot\|_{H^{\alpha}(\Omega)}$ and $\|\cdot\|_{H_{0}^{\alpha}(\Omega)}$ are equivalent in $H_{0}^{\alpha}(\Omega)$.

Proof. For $C^{2}$ bounded domain and $\alpha \in\left(\frac{1}{2}, 1\right)$, it follows by [12, Theorem 1.1] that

$$
\int_{\Omega} \frac{|u(x)|^{2}}{\rho^{2 \alpha}(x)} d x \leq c_{41} \int_{\Omega} \int_{\Omega} \frac{[u(x)-u(y)]^{2}}{|x-y|^{N+2 \alpha}} d x d y, \quad \forall u \in C_{c}^{2}(\Omega),
$$

which implies that

$$
\|u\|_{L^{2}(\Omega)} \leq c_{42}\|u\|_{H_{0}^{\alpha}(\Omega)}, \quad \forall u \in C_{c}^{2}(\Omega) .
$$

Since $C_{c}^{2}(\Omega)$ is dense in $H_{0}^{\alpha}(\Omega)$, then (3.10) holds in $H_{0}^{\alpha}(\Omega)$. We omit the left proof.

Proof of Theorem 1.1 part $(i)$. Uniqueness. Let $u, w$ be two weak solutions of (1.1), then we derive that

$$
\langle u-w, v\rangle_{H_{0}^{\alpha}(\Omega)}=0, \quad \forall v \in H_{0}^{\alpha}(\Omega) .
$$

Taking $v=u-w \in H_{0}^{\alpha}(\Omega)$, we have that

$$
\|u-w\|_{H_{0}^{\alpha}(\Omega)}=0 .
$$

Then we obtain the uniqueness.

Existence. Let $\left\{f_{n}\right\}_{n}$ be a sequence of functions in $\mathbb{X}_{\alpha}(\Omega)$ satisfying

$$
\lim _{n \rightarrow \infty}\left\|f_{n}-f\right\|_{L^{2}(\Omega)}=0 .
$$

Let $u_{n}$ be the classical solution of (1.1) with nonhomogeneous term $f_{n}$. Then

$$
\left\langle u_{n}, v\right\rangle_{H_{0}^{\alpha}(\Omega)}=\int_{\Omega} v f_{n} d x, \quad \forall v \in H_{0}^{\alpha}(\Omega)
$$

From Theorem 3.1, Proposition 3.2 and Hölder inequality, we have that

$$
\begin{aligned}
\left\|u_{n}\right\|_{H_{0}^{\alpha}(\Omega)}^{2}=\int_{\Omega} f_{n} u_{n} d x & \leq\left\|u_{n}\right\|_{L^{2}(\Omega)}\left\|f_{n}\right\|_{L^{2}(\Omega)} \\
& \leq c_{43}\left\|u_{n}\right\|_{H_{0}^{\alpha}(\Omega)}\left\|f_{n}\right\|_{L^{2}(\Omega)} .
\end{aligned}
$$

Then we have that

$$
\left\|u_{n}\right\|_{H_{0}^{\alpha}(\Omega)} \leq c_{43}\left\|f_{n}\right\|_{L^{2}(\Omega)} \leq c_{44}\|f\|_{L^{2}(\Omega)}^{2} .
$$

From [10, Theorem 6.7, Theorem 7.1], the embedding: $H_{0}^{\alpha}(\Omega) \hookrightarrow L^{2}(\Omega)$ is compact, then up to subsequence, there exists $u \in L^{2}(\Omega)$ such that

$$
u_{n} \rightarrow u \text { in }\left(H_{0}^{\alpha}(\Omega)\right)^{\prime} \quad \text { as } n \rightarrow \infty
$$

and

$$
\lim _{n \rightarrow \infty}\left\|u_{n}-u\right\|_{L^{2}(\Omega)}=0
$$


Then from (3.11), we have that

$$
\langle u, v\rangle_{H_{0}^{\alpha}(\Omega)}=\int_{\Omega} v f d x, \quad \forall v \in H_{0}^{\alpha}(\Omega),
$$

that is, $u$ is a weak solution of (1.1). Taking $v=u$ above, we deduce (1.2).

\section{VERY WEAK SOLUTION}

4.1. The case that $f \in L^{1}\left(\Omega, \rho^{\beta} d x\right)$. In this section, we may weaken the nonhomogeneous term $f$ to $L^{1}\left(\Omega, \rho^{\beta} d x\right)$ in $(1.1)$.

Proof of Theorem 1.1 part $(i i)$ when $f \in L^{1}\left(\Omega, \rho^{\beta} d x\right)$. Uniqueness. Let $u, w$ be two very weak solutions of (1.1), then

$$
\int_{\Omega}(u-w)(-\Delta)_{\Omega}^{\alpha} v d x=0, \quad \forall v \in \mathbb{X}_{\alpha}(\Omega) .
$$

Let $\eta_{u-w}$ be the solution of

$$
\left\{\begin{aligned}
(-\Delta)_{\Omega}^{\alpha} \eta_{u-w} & =\operatorname{sign}(u-w) & & \text { in } \quad \Omega, \\
\eta_{u-w} & =0 & & \text { on } \partial \Omega .
\end{aligned}\right.
$$

We observe that $\eta_{u-w} \in \mathbb{X}_{\alpha}(\Omega)$ and put $v=\eta_{u-w}$ in (4.1), then we obtain that

$$
\int_{\Omega}|u-w| d x=0
$$

which implies the uniqueness.

Existence. We make use of Proposition 3.1 to approximate the solution $u$ of (1.1) by a sequence of classical solutions. In fact, we choose a sequence of $C^{2}(\Omega) \cap C(\bar{\Omega})$ functions $\left\{f_{n}\right\}_{n}$ such that

$$
\lim _{n \rightarrow \infty}\left\|f_{n}-f\right\|_{L^{1}\left(\Omega, \rho^{\beta} d x\right)}=0 .
$$

Denote $u_{n}$ the solution of (1.1) with nonhomogeneous nonlinearity $f_{n}$. Then from Proposition 3.1, we have that

$$
\int_{\Omega} u_{n}(-\Delta)_{\Omega}^{\alpha} v d x=\int_{\Omega} f_{n}(x) v(x) d x, \quad \forall v \in \mathbb{X}_{\alpha}(\Omega) .
$$

By Lemma 2.5, it deduces that

$$
|v(x)| \leq c_{45} \rho^{\beta}(x), \quad \forall x \in \Omega
$$

and together with the convergence of $\left\{f_{n}\right\}_{n}$ in $L^{1}\left(\Omega, \rho^{\beta} d x\right)$, we obtain that

$$
\lim _{n \rightarrow \infty} \int_{\Omega} f_{n}(x) v(x) d x=\int_{\Omega} f(x) v(x) d x .
$$

For any $n, m \in \mathbb{N}$, let $\eta_{u_{m}-u_{n}}$ be the solution of (4.2) with nonhomogeneous term $\operatorname{sign}\left(u_{m}-u_{n}\right)$, then we obtain that

$$
\int_{\Omega}\left|u_{m}-u_{n}\right| d x=\int_{\Omega}\left(f_{m}-f_{n}\right) \eta_{u_{m}-u_{n}} d x \leq c_{46} \int_{\Omega}\left|f_{m}-f_{n}\right| \rho^{\beta} d x .
$$

For any $\epsilon>0$, it infers by (4.3) that there exists $N_{\epsilon}>0$ such that for any $n, m>N_{\epsilon}$,

$$
c_{47} \int_{\Omega}\left|f_{m}-f_{n}\right| \rho^{\beta} d x \leq \epsilon
$$

which implies that for any $n, m>N_{\epsilon}$

$$
\int_{\Omega}\left|u_{m}-u_{n}\right| d x \leq \epsilon
$$


Thus, $\left\{u_{n}\right\}_{n}$ is a Cauchy sequence in $L^{1}(\Omega)$ and then there exists $u \in L^{1}(\Omega)$ such that

$$
\lim _{n \rightarrow \infty}\left\|u_{n}-u\right\|_{L^{1}(\Omega)}=0 .
$$

Passing to the limit of (4.4) as $n \rightarrow \infty$, we obtain that

$$
\int_{\Omega} u(x)(-\Delta)_{\Omega}^{\alpha} v(x) d x=\int_{\Omega} f(x) v(x) d x, \quad \forall v \in \mathbb{X}_{\alpha}(\Omega) .
$$

Therefore, problem (1.1) has a very weak solution, that is,

$$
\int_{\Omega} u(-\Delta)_{\Omega}^{\alpha} v d x=\int_{\Omega} f v d x, \quad \forall v \in \mathbb{X}_{\alpha}(\Omega)
$$

choosing $v=\eta_{u}$, the solution of (4.2) with nonhomogeneous term $\operatorname{sign}(u)$, it infers from (4.7) that

$$
\int_{\Omega}|u| d x \leq c_{48} \int_{\Omega}|f| \rho^{\beta} d x
$$

The proof ends.

4.2. The case that $f \in \mathcal{M}\left(\Omega, \rho^{\beta}\right)$. In this subsection, we may weaken the nonhomogeneous term $f$ to $\mathcal{M}\left(\Omega, \rho^{\beta}\right)$ in (1.1).

Proof of Theorem 1.1 part $(i i)$ when $f \in \mathcal{M}\left(\Omega, \rho^{\beta}\right)$. Uniqueness. Let $u, w$ be two very weak solutions of (1.1), then

$$
\int_{\Omega}(u-w)(-\Delta)_{\Omega}^{\alpha} v d x=0, \quad \forall v \in \mathbb{X}_{\alpha}(\Omega),
$$

which reduces to $(4.1)$.

Existence. We make use of Proposition 3.1 to approximate the solution $u$ of (1.1) by a sequence of classical solutions. In fact, we choose a sequence of $C^{2}(\Omega) \cap C(\bar{\Omega})$ functions $\left\{f_{n}\right\}_{n}$ such that

$$
\lim _{n \rightarrow \infty} \int_{\Omega} f_{n} v d x=\int_{\Omega} v d f \text { for any } v \in \mathbb{X}_{\alpha}(\Omega) .
$$

Denote $u_{n}$ the solution of (1.1) with nonhomogeneous nonlinearity $f_{n}$. Then from Proposition 3.1 , we have that

$$
\int_{\Omega} u_{n}(-\Delta)_{\Omega}^{\alpha} v d x=\int_{\Omega} f_{n}(x) v(x) d x, \quad \forall v \in \mathbb{X}_{\alpha}(\Omega) .
$$

Thus, it deduces by (4.5) and (4.8) that

$$
\lim _{n \rightarrow \infty} \int_{\Omega} v(x) f_{n}(x) d x=\int_{\Omega} v(x) d f(x)
$$

To prove that $\left\{u_{n}\right\}_{n}$ is uniformly bounded in $L^{1}(\Omega)$. For any $n \in \mathbb{N}$, let $\eta_{u_{n}}$ be the solution of (4.2) with nonhomogeneous term $\operatorname{sign}\left(u_{n}\right)$, then we derive that

$$
\begin{aligned}
\int_{\Omega}\left|u_{n}\right| d x & =\int_{\Omega} f_{n} \eta_{u_{n}} d x \leq c_{49} \int_{\Omega}\left|\eta_{u_{n}}\right| d|f| \\
& \leq c_{49} c_{48}\left\|\eta_{u_{n}} \rho^{-\beta}\right\|_{L^{\infty}(\Omega)} \int_{\Omega} \rho^{\beta} d|f| \leq c_{50} \int_{\Omega} \rho^{\beta} d|f|,
\end{aligned}
$$

where $c_{49}, c_{50}>0$ are independent of $n$, since $\left|\eta_{u_{n}}\right| \leq c_{48} \rho^{\beta}$ in $\Omega$. 
To prove that $\left\{u_{n}\right\}_{n}$ is uniformly integrable. Let $\mathcal{O}$ be any Borel subset of $\Omega$, take $\eta_{\mathcal{O}}$ be the solution of $(4.2)$ with nonhomogeneous term $\chi_{\mathcal{O}} \operatorname{sign}(\mathcal{O})$, then we see that

$$
\begin{aligned}
\int_{\mathcal{O}}\left|u_{n}\right| d x & =\int_{\Omega} f_{n} \eta_{\mathcal{O}} d x \leq c_{49} \int_{\Omega}\left|\eta_{\mathcal{O}}\right| d|f| \\
& \leq c_{49} c_{48}\left\|\eta_{\mathcal{O}} \rho^{-\beta}\right\|_{L^{\infty}(\Omega)} \int_{\Omega} \rho^{\beta} d|f| \leq c_{51} \int_{\Omega} \rho^{\beta} d|f|
\end{aligned}
$$

where $c_{51}>0$ is independent of $n$. We observe that

$$
\begin{aligned}
\left|\rho^{-\beta}(x) \eta_{\mathcal{O}}(x)\right| & =\rho^{-\beta}(x)\left|\int_{\Omega} G_{\Omega, \alpha}(x, y) \chi_{\mathcal{O}}(y) \operatorname{sign}\left(u_{n}\right)(y) d y\right| \\
& \leq c_{7} \int_{\mathcal{O}} \frac{\rho^{\beta}(y)}{|x-y|^{N-2+2 \alpha}} d y \\
& \leq c_{7} D_{0}^{\beta} \int_{B_{d_{0}}(x)} \frac{1}{|x-y|^{N-2+2 \alpha}} d y \\
& \leq c_{52} d_{0}^{2-2 \alpha}=c_{52}|\mathcal{O}|^{\frac{2-2 \alpha}{N}}
\end{aligned}
$$

where $c_{52}>0$ is independent of $n, D_{0}=\sup _{x, y \in \omega}|x-y|$ and $d_{0}>0$ satisfying

$$
|\mathcal{O}|=\left|B_{d_{0}}(0)\right| \text {. }
$$

Thus, we derive that

$$
\int_{\mathcal{O}}\left|u_{n}\right| d x \leq c_{53}\|f\|_{\mathcal{M}_{\alpha}(\Omega)}|\mathcal{O}|^{\frac{2-2 \alpha}{N}}
$$

where $c_{53}>0$ is independent of $n$.

Therefore, we conclude that $\left\{u_{n}\right\}_{n}$ is uniformly bounded in $L^{1}(\Omega)$ and uniformly integrable, thus weakly compact in $L^{1}(\Omega)$ by the Dunford-Pettis Theorem, and there exists a subsequence $\left\{u_{n_{k}}\right\}_{k}$ and an integrable function $u$ such that $u_{n_{k}} \rightarrow u$ weakly in $L^{1}(\Omega)$. Passing to the limit in (4.9), we obtain that $u$ is a very weak solution of (1.1).

Choosing $v=\eta_{u}$, the solution of (4.2) with nonhomogeneous term $\operatorname{sign}(u)$, it infers that

$$
\int_{\Omega}|u| d x \leq c_{54} \int_{\Omega} \rho^{\beta} d|f|
$$

This ends the proof.

\section{General boundary data}

In this section, we consider the classical solution of (1.5) under the general boundary data. In [15, Theorem $1.3(i)$ ], the author proved the Integral by Part Formula

$$
\int_{\Omega} u(-\Delta)_{\Omega}^{\alpha} v d x=\int_{\Omega} v(-\Delta)_{\Omega}^{\alpha} u d x+\int_{\partial \Omega} v \frac{\partial^{\beta} u}{\partial \vec{n}^{\beta}} d \omega-\int_{\partial \Omega} u \frac{\partial^{\beta} v}{\partial \vec{n}^{\beta}} d \omega, \quad \forall u, v \in \mathbb{D}_{\beta},
$$

where $\mathbb{D}_{\beta}$ is given by (1.7).

However, it is open to show that the solution $u_{f, g}$ of $(1.5)$ belongs to $\mathbb{D}_{\beta}$, even under the hypothesis that

$$
f \in C^{2}(\Omega) \cap C(\bar{\Omega}) \quad \text { and } \quad g \in C^{2}(\partial \Omega) .
$$

Proposition 5.1. Assume that $f \in C^{2}(\Omega) \cap C(\bar{\Omega}), g \in C^{2}(\partial \Omega)$ and $u$ be the classical solution of (1.5). Then

$$
\int_{\Omega} u(-\Delta)_{\Omega}^{\alpha} v d x=\int_{\Omega} f v d x+\int_{\partial \Omega} g \frac{\partial^{\beta} v}{\partial \vec{n}^{\beta}} d \omega, \quad \forall v \in \mathbb{X}_{\alpha}(\Omega) \cap \mathbb{D}_{\beta} .
$$


Proof. Since $\Omega$ is a $C^{2}$ domain and $g \in C^{2}(\partial \Omega)$, then there exists a $C^{2}(\bar{\Omega})$ function $G$ such that

$$
G=g \quad \text { on } \quad \partial \Omega .
$$

Let $u_{g}$ be the solution of $(1.5)$ and denote

$$
u_{0}=u_{g}-G \text {. }
$$

Then $u_{0}$ satisfies (2.15). From [16, Proposition 2.3], $(-\Delta)_{\Omega}^{\alpha} G \in C_{l o c}^{2-2 \alpha}(\Omega)$ and

$$
\left|(-\Delta)_{\Omega}^{\alpha} G(x)\right| \leq c_{55} \rho(x)^{-\beta}, \quad \forall x \in \Omega .
$$

Choose $\tilde{g}_{n}$ a sequence of $C^{2}(\Omega) \cap C(\bar{\Omega})$ functions such that

$$
\lim _{n \rightarrow \infty}\left\|\left(\tilde{g}_{n}-(-\Delta)_{\Omega}^{\alpha} G\right) \rho^{\beta}\right\|_{L^{\infty}(\Omega)}=0
$$

Let $w_{n}$ be the solution of

$$
\left\{\begin{aligned}
(-\Delta)_{\Omega}^{\alpha} u & =f-\tilde{g}_{n} & & \text { in } \quad \Omega, \\
u & =0 & & \text { on } \quad \partial \Omega .
\end{aligned}\right.
$$

By Proposition 3.1, it infers that for $v \in \mathbb{X}_{\alpha}(\Omega)$,

$$
\begin{aligned}
\int_{\Omega} w_{n}(x)(-\Delta)_{\Omega}^{\alpha} v(x) d x & =\int_{\Omega} v(x)(-\Delta)_{\Omega}^{\alpha} w_{n}(x) d x \\
& =\int_{\Omega} v(x) f(x) d x-\int_{\Omega} v(x) \tilde{g}_{n} d x .
\end{aligned}
$$

We observe that

$$
\left\|w_{n}-u_{g}\right\|_{L^{\infty}(\Omega)} \leq c_{37}\left\|\left(\tilde{g}_{n}-(-\Delta)_{\Omega}^{\alpha} G\right) \rho^{\beta}\right\|_{L^{\infty}(\Omega)} .
$$

Therefore, passing to the limit of (5.5) as $n \rightarrow \infty$, we have that

$$
\int_{\Omega} u_{0}(x)(-\Delta)_{\Omega}^{\alpha} v(x) d x=\int_{\Omega} v(x) f(x) d x-\int_{\Omega} v(x)(-\Delta)_{\Omega}^{\alpha} G(x) d x .
$$

Since $G \in C^{2}(\bar{\Omega})$, then for $v \in \mathbb{D}_{\beta}$, it deduces by (5.1) that

$$
\int_{\Omega} v(x)(-\Delta)_{\Omega}^{\alpha} G(x) d x=\int_{\Omega} G(x)(-\Delta)_{\Omega}^{\alpha} v(x) d x-\int_{\partial \Omega} g \frac{\partial^{\beta} v}{\partial \vec{n}^{\beta}} d \omega .
$$

Thus,

$$
\int_{\Omega} u_{g}(-\Delta)_{\Omega}^{\alpha} v d x=\int_{\Omega}\left(u_{0}+G\right)(-\Delta)_{\Omega}^{\alpha} v d x=\int_{\Omega} v f d x+\int_{\partial \Omega} g \frac{\partial^{\beta} v}{\partial \vec{n}^{\beta}} d \omega .
$$

The proof ends.

Remark 5.1. The function space $C_{c}^{2}(\Omega)$ is a subset of $\mathbb{X}_{\alpha}(\Omega) \cap \mathbb{D}_{\beta}$, so $\mathbb{X}_{\alpha}(\Omega) \cap \mathbb{D}_{\beta}$ is not empty. However, $C_{c}^{2}(\Omega)$ is not proper for the Integral by Parts Formula since the boundary term $\int_{\partial \Omega} g \frac{\partial^{\beta} v}{\partial \vec{n}^{\beta}} d \omega$ always vanishes for $v \in C_{c}^{2}(\Omega)$.

Acknowledgements: H. Chen is supported by NSFC, No: 11401270, and by the Jiangxi Provincial Natural Science Foundation, No: 20161ACB20007 and the Project-sponsored by SRF for ROCS, SEM. H. Chen would like to thank professor Patricio Felmer for the supporting in the visiting CMM of University of Chile. 


\section{REFERENCES}

[1] Ph. Bénilan and H. Brezis, Nonlinear problems related to the Thomas-Fermi equation, J. Evolution Eq. 3, 673-770 (2003).

[2] P. Baras and M. Pierre, Critéres d'existence de solutions positives pour des équations semi-linéaires non monotones, Ann. Inst. H. Poincaré, Analyse Non Linéaire 2, 185-212 (1985).

[3] K. Bogdan, K. Burdzy and Z. Chen, Censored stable processes, Probability theory and related fields 127, 89-152 (2003).

[4] H. Brezis, Functional analysis, Sobolev spaces and partial differential equations, Springer Science $\mathcal{G}_{6}$ Business Media, (2010).

[5] Z. Chen and P. Kim, Green function estimate for censored stable processes, Probability theory and related fields 124, 595-610 (2002).

[6] Z. Chen, P. Kim and R. Song, Two-sided heat kernel estimates for censored stable-like processes, Probability theory and related fields 146, 361-399 (2010).

[7] H. Chen and L. Véron, Semilinear fractional elliptic equations involving measures, J. Diff. Eq. 25\%, 1457-1486 (2014).

[8] H. Chen and L. Véron, Semilinear fractional elliptic equations with gradient nonlinearity involving measures, J. Funct. Anal. 266, 5467-5492 (2014)

[9] H. Chen and L. Véron, Weakly and strongly singular solutions of semilinear fractional elliptic equations, Asym. Anal. 88, 165-184 (2014).

[10] E. Di Nezza, G.Palatucci and E. Valdinoci, Hitchhiker's guide to the fractional Sobolev spaces, Bull. Sci. Math. 136 (5), 521-573 (2012).

[11] J. Duan, An Introduction to Stochastic Dynamics, Cambridge University Press, New York (2015).

[12] B. Dyda, A fractional order Hardy inequality, Illinois J. Math. 48, 575-588 (2004).

[13] M. Fukushima, Y. Ōshima and M. Takeda, Dirichlet forms and symmetric Markov processes, Walter de Gruyter 19, (2010).

[14] A.Gmira and L. Véron, Boundary singularities of solutions of some nonlinear elliptic equations, Duke Math. J. 64, 271-324 (1991).

[15] Q. Guan, Integration by parts formula for regional fractional laplacian, Comm. Math. Phys. 266, 289-329 (2006).

[16] Q. Guan and Z. Ma, Reflected symmetric -stable processes and regional fractional Laplacian, Probability theory and related fields 134, 649-694 (2006).

[17] J. Klafter, M. Shlesiger and G. Zumofen, Beyond Brownian motion, Physics Today, 49, 33-39 (1996).

[18] Y. Li and L. Nirenberg, The Dirichlet problem for singularly perturbed elliptic equations, Comm. Pure Appl. Math. 51, 1445-1490 (1998).

[19] R. Metzler and J. Klafter, The Random Walks Guide to Anomalous Diffusion: A Fractional Dynamics Approach, Phys. Rep. 339, 1-77 (2000).

[20] A. C. Ponce, Selected problems on elliptic equations involving measures, arXiv: 1204.0668 (2012).

[21] T. Solomon, E. Weeks and K. Swinney, Observation of anomalous diffusion and Lévy flights in a twodimensional rotating flow, Phys. Rev. Lett. 71, 3975-3978 (1993).

[22] X. Ros-Oton and J. Serra, The Dirichlet problem for the fractional laplacian: regularity up to the boundary, J. Math. Pures Appl. 101, 275-302 (2014).

[23] L. Véron, Elliptic equations involving Measures, Stationary Partial Differential equations, Vol. I, 593712, Handb. Differ. Equ., North-Holland, Amsterdam (2004). 